\title{
Particle-laden tubeless siphon
}

\author{
By J. WA NG AND D. D. JOSEPH \\ Department of Aerospace and Engineering Mechanics, University of Minnesota, MN 55455, USA
}

(Received 26 September 2002 and in revised form 2 January 2003)

A tubeless siphon was created by sucking a $1 \%$ aqueous Polyox $\dagger$ solution laden with particles from a beaker into a cylinder by moving a piston. The piston speed and particle concentration were varied. At very high rates of withdrawal, all the liquid could be removed before the siphon broke. In this case, the beaker was completely cleaned without a trace of liquid. The addition of small concentrations of small, nearly neutrally buoyant particles greatly enhanced the pulling power of the liquid, reducing the threshold speed of withdrawal at which the beaker was completely cleaned. At speeds of withdrawal smaller than the threshold not all of the fluid-particle mixture is pulled out of the beaker. The amount pulled out first increases, then decreases as the particle concentration is increased.

\section{Introduction}

The tubeless siphon is a well-known special effect found in polymeric liquids and not in Newtonian liquids. This effect is well documented and is described in most of the standard works on rheological fluid mechanics, e.g. Bird, Armstrong \& Hassager (1977), Joseph (1990), and Macosko (1994). The effect may be described as follows: a fluid is sucked through a nozzle elevated above the surface of the liquid, as shown in figure 1. Instead of the liquid breaking as for a Newtonian liquid, an unsupported fluid column is drawn from a pool below into the nozzle above without breaking, as shown in figure 2.

Boonacker (1999) studied the screening properties of particle-laden viscoelastic fluids, using the apparatus, fluid and particles used in this study. She reported that when the suction speed was sufficiently high, only the fluid would enter the tube; the particles were filtered out as in the Uebler effect. This filtering is called a screening effect. The tubeless siphon with particles was observed in her experiments.

Here we make a systematic study of the tubeless siphon with particles, pointing out the previously undocumented 'cleaning property' which may have applications for decontamination. At a threshold withdrawal speed of the piston, the tubeless siphon laden with particles leads to complete removal of all fluid and particles from the pool (figure $2 a$ ). If there are no particles in the fluid, or the concentration of particles is lower than a critical value, the fluid column breaks during siphoning leaving a measurable volume of liquid behind (figure $2 b$ ). Movies and photos of the experiments reported in this paper can be viewed at http://www.aem.umn.edu/ research/particles/siphon/. 


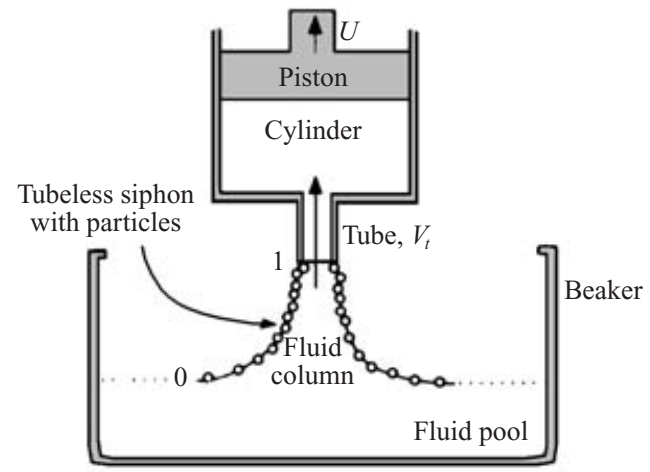

FIgURE 1. A schematic of the experimental set up.

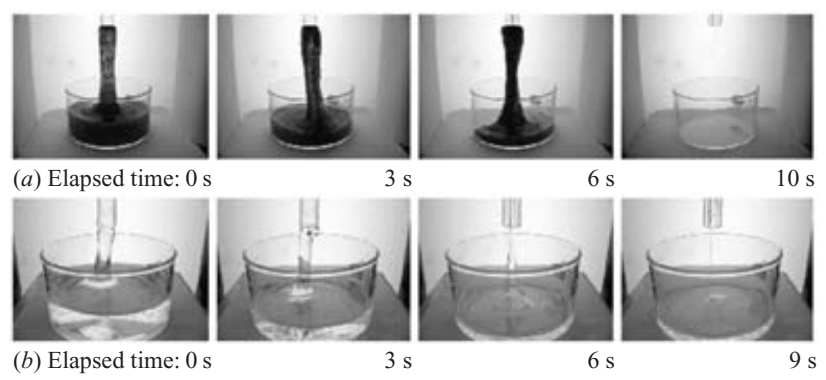

Figure 2. Sequence of photos of a tubeless siphon of $1 \%$ aqueous Polyox solution at a piston speed of $0.224 \mathrm{~cm} \mathrm{~s}^{-1}$. (a) The siphon is loaded with about $15 \%$ by volume of $600-700 \mu \mathrm{m}$ resin particles. All the mixture is cleaned from the beaker. $(b)$ When there are no particles, fluid is left in the beaker. The same container is used in $(a)$ and $(b)$; the initial volume of the fluid-particle mixture in $(a)$ is equal to the initial volume of the fluid in $(b)$.

\section{Experimental conditions}

The apparatus used in the experiment is shown schematically in figure 1 ; it consists of three primary parts: a piston pump, a tube and a beaker. Pumping takes place in a $8.89 \mathrm{~cm}$ long cylinder with $10.08 \mathrm{~cm}$ inner diameter and a piston driven by an Instron tensile machine. A tube with $1.27 \mathrm{~cm}$ outer diameter and $0.470 \mathrm{~cm}$ inner diameter is inserted into the cylinder from the bottom. The beaker is the reservoir for the viscoelastic fluid with particles.

The viscoelastic fluid used in the experiments was $1 \%$ by weight aqueous WSR 301 Polyox solution. Polyethene oxide has extremely long molecular chains with a molecular weight of $4 \times 10^{6} \mathrm{~g} \mathrm{~mol}^{-1}$, giving non-Newtonian and viscoelastic characteristics to the de-ionized water in which it has been dissolved. The process of dissolving Polyox powder into water was slow and it took about one week of continuous mixing to obtain one gallon of homogeneous solution.

Two kinds of particles were used in the experiments. Spherical, colourless, polymeric beads were used in most runs. The density of the beads was $1.0 \mathrm{~g} \mathrm{~cm}^{-3}$ and the size was in the range of $850-1000 \mu \mathrm{m}$. Spherical brown resin particles with sizes in the range of $600-700 \mu \mathrm{m}$ were also used, which are visible in photographs. The resin particles were nearly neutrally buoyant in $1 \%$ aqueous Polyox solution.

The following procedures were followed in the experiments. A well-mixed test fluid with suspended particles was prepared. At the beginning of each run, the piston was 


$$
\begin{array}{ccccc}
T\left({ }^{\circ} \mathrm{C}\right) & \rho\left(\mathrm{g} \mathrm{cm}^{-3}\right) & \eta_{0}(\mathrm{~Pa} \mathrm{~s}) & \psi_{10}\left(\mathrm{~g} \mathrm{~cm}^{-1}\right) & \lambda_{0}(\mathrm{~s}) \\
24 & 1 & 7.65 & 360 & 0.390
\end{array}
$$

TABLE 1. Key rheological parameters of $1 \%$ aqueous Polyox solution at $24{ }^{\circ} \mathrm{C} . T$ is the temperature; $\rho$ is the density; $\eta_{0}$ is the shear viscosity at zero shear rate; $\psi_{10}$ is the first normal stress coefficient at zero shear rate; $\lambda_{0}$ is the longest relaxation time.

at its lowest position in the cylinder. The beaker was lifted so that the tube dipped into the pool of liquid. The Instron tensile machine was started, raising the piston at a constant speed. The pressure difference between the atmosphere and the vacuum in the cylinder drove the fluid into the cylinder through the tube. Once the siphon started, the beaker was lowered so that the nozzle of the tube was higher than the fluid surface and the tubeless siphon formed. As the piston rose at a constant speed, the tubeless siphon could either suck all the liquid out of the beaker, or the siphon would break, leaving behind a measurable volume of liquid. The volume of the fluid which entered the cylinder was also recorded.

The distance from the nozzle to the surface of the pool at the moment when the tubeless siphon started was kept at $15 \mathrm{~cm}$ (with error less than $1 \mathrm{~cm}$ ). The temperatures under which the experiments were conducted were in the range of $21.1^{\circ} \mathrm{C}$ to $22.78^{\circ} \mathrm{C}$. Several key rheological parameters of $1 \%$ aqueous Polyox solution can be found in Liu (1995) (see table 1). However, extensional viscosities are not available.

\section{Analysis}

To investigate the properties of the particle-laden tubeless siphon, it is necessary to understand the mechanism of the breaking of the tubeless siphon. The volume flow rate in the cylinder and the tube is given by

$$
U A=V_{t} A_{t},
$$

where $U$ is the speed of the piston and $A$ is the inner cross-sectional area of the cylinder; $V_{t}$ is the velocity of the fluid in the tube and $A_{t}$ is the inner cross-sectional area of the tube (see figure 1). In each run, the piston speed $U$ was kept constant giving rise to a constant $V_{t}$; conservation of mass implies that

$$
V_{1} A_{1}=V_{0} A_{0},
$$

where $V_{1}$ and $A_{1}$ are the velocity and the cross-sectional area of the fluid column at the nozzle and $V_{0}$ and $A_{0}$ are the velocity and the cross-sectional area of the fluid column at the surface of the pool (figure 1).

Before the siphon breaks, $V_{1}$ and $A_{1}$ are balanced by $V_{t}$ and $A_{t}$. Therefore, during the process of siphoning, the volume flux of the fluid drawn out of the pool $\left(V_{0} A_{0}\right)$ is a constant. If the piston speed is high, this leads to a high extensional rate and high extensional force which can maintain a constant volume flux in the siphon until all the fluid is sucked out of the beaker. However, if the piston speed is low, the volume flux will decrease at some point in the siphoning process:

$$
V_{0} A_{0}=V_{1} A_{1}<V_{t} A_{t} .
$$

At that moment, $V_{1}$ is still equal to $V_{t}$, and so $A_{1}<A_{t}$. This inequality implies that air will be sucked into the tube and the pressure difference between the atmosphere 


$\begin{array}{lcccccccc}U\left(\mathrm{~cm} \mathrm{~s}^{-1}\right) & 0.0704 & 0.0895 & 0.109 & 0.128 & 0.147 & 0.166 & 0.185 & 0.205 \\ T\left({ }^{\circ} \mathrm{C}\right) & 21.83 & 22.61 & 22.50 & 22.44 & 22.00 & 21.94 & 21.94 & 21.94 \\ F & 0.0 & 0.0 & 0.033 & 0.067 & 0.167 & 0.33 & 0.80 & 1.00\end{array}$

TABLE 2. The suction fraction $F$ vs. the piston speed $U$ for Polyox solutions without particles. When $U$ is higher than the threshold $U_{T h}\left(0.185<U_{T h}<0.205 \mathrm{~cm} \mathrm{~s}^{-1}\right), F=1$ and all the fluid is removed from the beaker.

and the inside of the cylinder will vanish rapidly. Consequently, the tubeless siphon stops.

From the analysis and experiments, it is found that the cleaning properties depend on a number of conditions: the piston speed, the concentration of particles in the fluid, the properties of the fluid, the properties of the particles, the size and the shape of tube, and the distance of the nozzle from the pool. Moreover, temperature plays an important role because the properties of the Polyox solution are very sensitive to the temperature. This study will focus on the piston speed and the concentration of the particles in the fluid. The study of other conditions will be conducted in the future.

\section{Experimental results}

First we give results for the siphon of pure fluid and then for the siphon of the fluid-particle mixture.

\subsection{Pure fluid siphon}

The effect of the piston speed was studied in a series of runs using Polyox solutions without particles. In all these runs, the original volume of the Polyox solution in the beaker was $150 \mathrm{ml}$. The piston speed ranged from 0.0704 to $0.205 \mathrm{~cm} \mathrm{~s}^{-1}$. When the siphon stopped, the volume of the Polyox solution left in the beaker was recorded (the error was $\pm 5 \mathrm{ml}$ ). In table 2 , the piston speed $U$, the fluid temperature $T$, and the suction fraction $F$ (volume fraction sucked out of the original volume) are listed.

Table 2 indicates that the higher the piston speed, the more the fluid was sucked out. A higher piston speed leads to a higher extensional rate and larger extensional force which can pull more fluid out of the beaker. The threshold piston speed $U_{T h}$ for pure fluid siphon under our experimental conditions is between 0.185 and $0.205 \mathrm{~cm} \mathrm{~s}^{-1}$. The fluid is always completely removed when the piston speed is higher than the threshold.

When $U$ was 0.0704 or $0.0895 \mathrm{~cm} \mathrm{~s}^{-1}$, the volume of fluid sucked out was so small that it could not be measured reliably, hence $F$ was taken as zero in table 2 . If these two cases are excluded, the data can be described by a power law. $F$ vs. $U$ is plotted for $U=0.109,0.128,0.147,0.166,0.185$ and $0.205 \mathrm{~cm} \mathrm{~s}^{-1}$ in figure 3 ; a power law fits the data extraordinarily well:

$$
\left.\begin{array}{ll}
F=15600 U^{5.94} & \text { for } U<U_{T h} \\
F=1 & \text { for } U \geqslant U_{T h}
\end{array}\right\}
$$

where $0.185<U_{T h}<0.205 \mathrm{~cm} \mathrm{~s}^{-1}$.

Equation (4.1) shows that the piston speed has a large effect on the suction fraction because the suction fraction is proportional to the piston speed raised to the power 5.94. The prefactor and exponent in the power law correlation could be functions of other parameters, such as the polymer properties and the distance from the nozzle to 


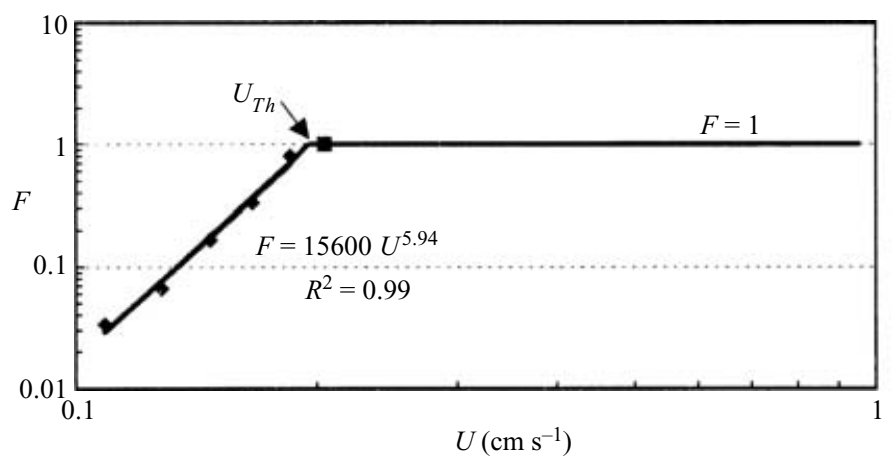

Figure 3. Power law fitting for $F$ vs. $U$. The fraction of fluid $F$ sucked out of the original volume increases with the piston speed $U$ raised to the power 5.94. For $U \geqslant U_{T h}\left(0.185<U_{T h}<0.205 \mathrm{~cm} \mathrm{~s}^{-1}\right)$ all of the fluid is removed from the beaker; hence $F=1$.

the pool. The threshold piston speed for a pure fluid siphon was lower when a Polyox solution with a molecular weight of $8 \times 10^{6} \mathrm{~g} \mathrm{~mol}^{-1}$ instead of $4 \times 10^{6} \mathrm{~g} \mathrm{~mol}^{-1}$ was used. We also believe that the threshold will be even lower if a solution with higher Polyox concentration is used. Systematic tests on $U$ vs. $F$ for different liquids have not yet been done. It may be possible to use the beaker test as a kind of viscometer for extensional flows.

\subsection{Particle-laden siphon}

The concentration $C$ of particles is given as a percentage of the total volume:

$$
C=\frac{\text { volume of particles }}{\text { volume of particles }+ \text { volume of fluid }} \times 100 \text {. }
$$

Tests of the effects of concentration were done by varying the concentration in siphon experiments at a fixed speed. Particles were added to $150 \mathrm{ml}$ of liquid to create increments of $C$. The mixture was stirred to be as homogenous as possible. To reduce the possibility of memory effects, the experiments were conducted at least $20 \mathrm{~s}$ after stirring. Since the longest relaxation time of the $1 \%$ aqueous Polyox solution is about $0.4 \mathrm{~s}$ (table 1), stirring should not have an appreciable effect on the test result. For each value of $C$ the volume left in the beaker after the siphon broke was recorded.

Experiments were carried out for $U=0.0704,0.0895,0.109,0.128,0.147$ and $0.166 \mathrm{~cm} \mathrm{~s}^{-1}$. The concentration of the particles ranged from $0.66 \%$ to $10 \%$. Studies of $F$ vs. $C$ for highly concentrated fluid-particle mixtures were not done. In table 3, the piston speed $U$, the mixture temperature $T$, and the suction fraction $F$ are listed. The data in table 3 show that for $U=0.109,0.128,0.147$ and $0.166 \mathrm{~cm} \mathrm{~s}^{-1}$, the presence of particles has dramatic affect on the suction fraction. At about $1 \%$ particle concentration, the mixture can be removed completely; without particles only a small fraction (less than $1 / 3$ ) of the fluid can be sucked out. Data in table 3 and figure 4 show that $F$ first increases and then decreases with $C$ for $U=0.0704$ and $0.0895 \mathrm{~cm} \mathrm{~s}^{-1}$; complete cleaning was not observed at these speeds.

The threshold piston speed $U_{T h}$ at which complete cleaning is obtained depends on the particle concentration. As shown in $\S 4.1,0.185<U_{T h}<0.205 \mathrm{~cm} \mathrm{~s}^{-1}$ for a pure fluid siphon $(C=0)$. The presence of particles reduces the threshold value, i.e. $U_{T h}(C>0)<U_{T h}(C=0)$. For example, table 3 shows that when $C \%=0.99 \%$, the threshold value reduces to $0.109<U_{T h}<0.128 \mathrm{~cm} \mathrm{~s}^{-1}$. However, there exists 


\begin{tabular}{|c|c|c|c|c|c|c|c|}
\hline$U\left(\mathrm{~cm} \mathrm{~s}^{-1}\right)$ & $T\left({ }^{\circ} \mathrm{C}\right)$ & $C(\%)$ & $F$ & $U\left(\mathrm{~cm} \mathrm{~s}^{-1}\right)$ & $T\left({ }^{\circ} \mathrm{C}\right)$ & $C(\%)$ & $F$ \\
\hline \multirow[t]{8}{*}{0.0704} & 21.83 & 0 & 0 & 0.109 & 22.5 & 0 & 0.033 \\
\hline & & 0.99 & 0.175 & & & 0.99 & 0.967 \\
\hline & & 1.96 & 0.183 & & & 1.96 & 1.000 \\
\hline & & 2.98 & 0.191 & 0.128 & 22.44 & 0 & 0.067 \\
\hline & & 4.00 & 0.200 & 0.120 & & 0.99 & 1.000 \\
\hline & & $\begin{array}{l}5.00 \\
6.02\end{array}$ & $\begin{array}{l}0.430 \\
0248\end{array}$ & & & 1.96 & 1.000 \\
\hline & & $\begin{array}{l}0.02 \\
7.01\end{array}$ & $\begin{array}{l}0.240 \\
0.194\end{array}$ & 0.147 & 22.0 & 0 & 0.167 \\
\hline & & 10.02 & 0.190 & & & 0.99 & 1.00 \\
\hline \multirow{7}{*}{0.0895} & 22.61 & 0 & 0 & & & 1.96 & 1.00 \\
\hline & & 0.99 & 0.670 & & & 2.98 & 1.00 \\
\hline & & 1.96 & 0.869 & & & 5.00 & 1.00 \\
\hline & & 2.98 & 0.871 & & & 10.02 & 1.00 \\
\hline & & 4.00 & 0.712 & 0.166 & 21.94 & 0 & 0.333 \\
\hline & & 5.00 & 0.525 & & & 0.66 & 1.00 \\
\hline & & 7.01 & 0.225 & & & 5.00 & 1.00 \\
\hline
\end{tabular}

TABLE 3. The suction fraction $F$ vs. the concentration of particles $C$. Piston speed $U=0.0704,0.0895,0.109,0.128,0.147$ and $0.166 \mathrm{~cm} \mathrm{~s}^{-1}$.

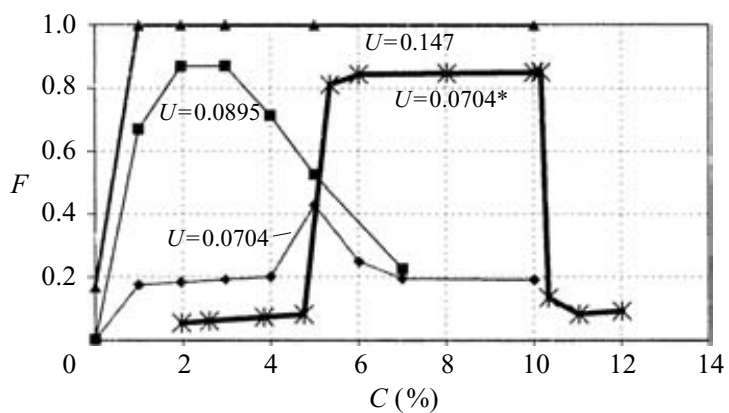

Figure 4. The suction fraction $F$ vs. the concentration of particles $C \%<12 \%$. Piston speed $U=0.0704,0.0895$ and $0.147 \mathrm{~cm} \mathrm{~s}^{-1}$. Data are from table 3 . The plot denoted by $U=0.0704 *$ is for experiments at piston speed $U=0.0704 \mathrm{~cm} \mathrm{~s}^{-1}$ using a degraded Polyox solution stored in a refrigerator for six months.

a minimum threshold speed $\tilde{U}_{T h}$ so that when $U<\tilde{U}_{T h}$, complete cleaning cannot be achieved no matter what the particle concentration $C$ is. Table 3 shows that the minimum threshold $\tilde{U}_{T h}$ is between 0.0895 and $0.109 \mathrm{~cm} \mathrm{~s}^{-1}$ in our experiments.

A critical concentration could be defined as the smallest concentration of particles at which the suction fraction $F$ reaches its peak value under a fixed $U$. For $U=0.0704$ and $0.0895 \mathrm{~cm} \mathrm{~s}^{-1}$, the critical $C$ is that at which $F$ peaks. For $U=0.109,0.128,0.147$ and $0.166 \mathrm{~cm} \mathrm{~s}^{-1}$, the critical $C$ is the smallest $C$ at which the complete removal is obtained. For $U=0.205 \mathrm{~cm} \mathrm{~s}^{-1}$, fluid without particles can be completely removed; hence, the critical $C$ is 0 . The critical $C$ for each value of $U$ is listed in table 4 and plotted in figure 5. The critical $C$ decreases as $U$ increases.

It is difficult to accurately determine the critical concentration mainly because the Polyox solution degrades very quickly after its creation and is also very sensitive to temperature. For these reasons, the precise values of the suction fraction are erratic and not easily reproduced. We performed certain experiments to verify the existence 


$\begin{array}{llllllll}U\left(\mathrm{~cm} \mathrm{~s}^{-1}\right) & 0.0704 & 0.0895 & 0.109 & 0.128 & 0.147 & 0.166 & 0.205 \\ \text { Critical } C(\%) & 5.00 & 2.98 & 1.96 & 0.99 & 0.99 & 0.66 & 0.00\end{array}$

TABLE 4. The critical $C$ for each value of $U$.

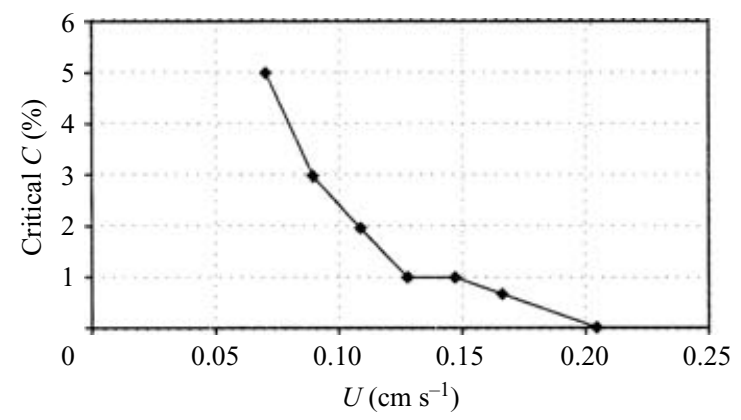

FIgURE 5. The critical $C$ vs. $U$.

rather than the precise value of a critical concentration of particles at piston speed below the threshold speed. To test the effects of degradation, we performed experiments using two samples of the same Polyox solution; one was tested immediately after its creation and the other after six months of storage in a refrigerator. We believe that the refrigerated solution degraded by oxidation (see Bailey \& Koleske 1976). The $F$ vs. $C$ graph for the degraded solution is compared with the fresh solution at $U=0.0704 \mathrm{~cm} \mathrm{~s}^{-1}$ in figure 4 . The critical concentration of particles for the degraded solution is higher than that for the fresh solution as would be expected; the maximum $F$ for the degraded solution is higher than that for the fresh solution, probably because the test temperature for the degraded solution is $0.9-1.2^{\circ} \mathrm{C}$ lower than for the fresh solution. Another difference is that the $F$ vs. $C$ curve near the criticality for the fresh solution is more sharply peaked than for the degraded solution. It is probable that the $F$ vs. $C$ curves would be smooth in experiments in very finely divided intervals of $C$.

\section{Mechanics of the particle-laden tubeless siphon}

In this section, we propose some explanations of the mechanics of the particle-laden tubeless siphon. When the tubeless siphon starts, the particles which are sucked up in the fluid column appear to be trapped at the free surface of the siphon, with few or no particles inside. Such a distribution of particles is related to the velocity profile in the fluid column. According to Matthys (1988), the axial velocity at the centreline is much greater than the velocity of the free surface at the same axial location (see figure 6). The velocity profile is similar to the velocity profile in Poiseuille flow in a tube. However, there is no wall in the siphon flow, hence no lubrication force to push the particles away from the free surface, and strong return flow at the free surface driven by gravity can be observed. As a result, most of the particles are driven away from the centreline to the free surface.

Figure 7 shows a sequence of photos for one siphon experiment using brown resin particles. In figure $7(a, b)$, it can be seen that particles are brought up by the siphon but filtered out at the nozzle; almost none of them enter the tube. In experiments, 


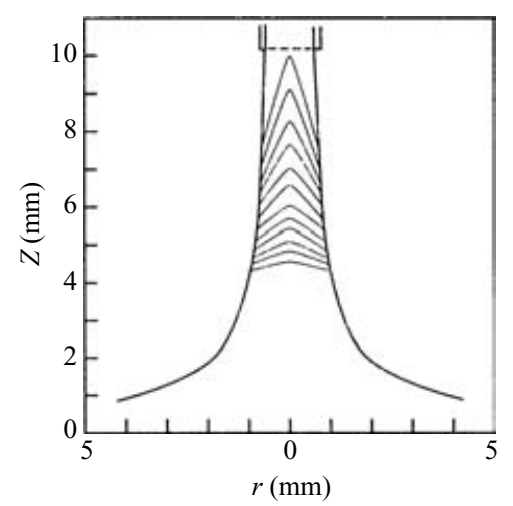

Figure 6. The velocity profile in the fluid column in tubeless siphon according to Matthys (1988). In the experiments a gravity-driven return flow at the free surface makes the difference in velocity between centreline and free surface even greater.

(a)

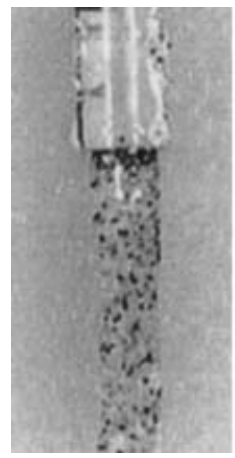

(b)

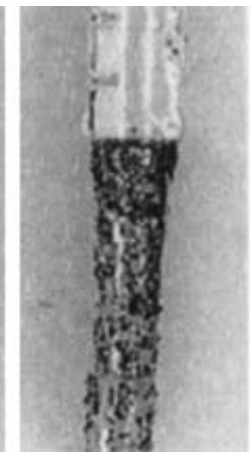

(c)

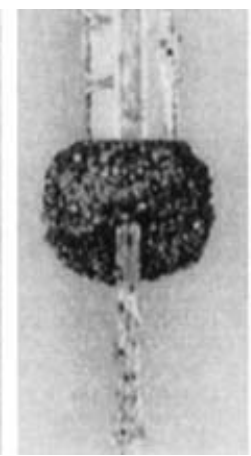

(d)

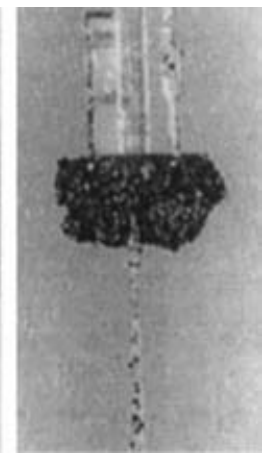

FIGURE 7. Photos for siphon experiments using brown resin particles. In $(a)$ and $(b)$ nearly all the particles are filtered out and do not enter the nozzle. In $(c)$ and $(d)$ particles cluster around the nozzle and the siphon continues even though the fluid column has a smaller radius than the tube. From Boonacker (1999).

we noted that particles screened from entering the tube fall in a return flow on the outside of the siphon. If the concentration of the particles is sufficiently high, the particles gather at the free surface of the fluid column, forming a pseudo-tube from the nozzle to the pool (see figure $2 a$ ). If the concentration of the particles is lower, they cannot cover the free surface of the whole column. However, in the portion adjacent to the nozzle, due to the screening effects which will be discussed next, the surface of the column is almost always covered by particles (see figure 7). These particles apparently serve as an extension of the tube. The particles at the free surface could provide additional support for the tubeless siphon and improve the suction.

The screening effect reported in Boonacker (1999) plays an important role in the enhancement of the suction. The screening effect means that when the suction speed is sufficiently high, only the fluid enters the tube and the particles are filtered out. This effect is closely related to the characteristics of the entrance flow of viscoelatic fluids (Binding \& Walters 1988). Figure 8 illustrates such characteristics schematically in comparison with the injection flow of Newtonian fluids. Suction and injection are asymmetric affects and the asymmetry has opposite polarity in Newtonian and 


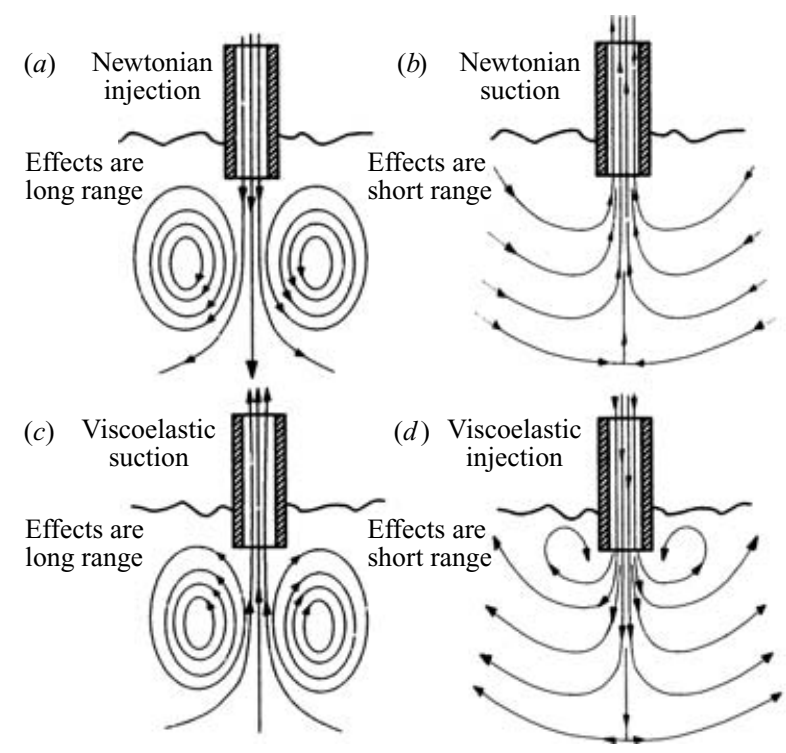

FIGURE 8 . Asymmetry of suction and injection in Newtonian and viscoelastic fluid (they are just the opposite).

viscoelastic fluids. In Newtonian fluids, injection is a long-range effect associated with the propagation of vorticity created at the nozzle exit as shown in figure $8(a)$. The flow into a nozzle does not generate vorticity and the streamline are like potential sink flow (figure $8 b$ ). One familiar consequence of the asymmetry of suction and injection in Newtonian fluids is that it is easy to blow out a candle even from great distances but it is nearly impossible to suck it out. The circulations which develop in viscoelastic fluid are opposite to those in Newtonian fluid; all the eddies are reversed. The secondary motion which develops in suction draws fluid remotely into the nozzle as shown in figure $8(c)$. The circulation which develops when a viscoelastic fluid is injected from a nozzle into a reservoir (figure $8 d$ ) is opposite to that shown for suction (figure $8 c$ ); it inhibits long-range injection effects and tends to transfer fluid sideways in a motion similar to source flow with streamlines reversed.

The higher the suction speed, the stronger the circulation. In the siphon flow near the nozzle, the particles at the free surface of the fluid column are trapped in the circulation and cannot enter the nozzle. As a result, more and more particles accumulate near the nozzle and cover the upper portion of the fluid column. Due to this accumulation, a cluster of particles can often be seen around the nozzle (see figure $7 c, d$ ). When the volume of fluid left in the beaker decreases, the fluid column will 'neck' at the nozzle so that the cross-sectional area of the liquid column $A_{1}$ is smaller than the cross-sectional area of the tube $A_{t}$; air will be sucked in and the liquid column ruptures, stopping the siphon. However, the presence of the cluster of particles around the nozzle can prevent air from entering the nozzle. The fluid column shown in figure $7(c, d)$ has a smaller radius than the tube, but the siphon does not stop. Thus, the presence of the particles prolongs the time of the siphon process and helps the suction.

When $U=0.0704$ and $0.0895 \mathrm{~cm} \mathrm{~s}^{-1}$, increasing the concentration of particles beyond the critical value of $C$ caused a decrease of the suction fraction $F$. The reason for this is not understood. 


\section{Conclusion}

The new results reported here are:

(a) The pulling power of the tubeless siphon of a $1 \%$ aqueous Polyox solution is greatly increased by the suction speed. When there are no particles in the fluid, the suction fraction is proportional to the suction speed raised to the power 5.94.

(b) At sufficiently high rates of withdrawal all the liquid may be removed by the siphon, leaving behind a dry and clean beaker. We call this a cleaning property of the tubeless siphon.

(c) The pulling power of a siphon is dramatically increased by the presence of small particles in concentrations less than $10 \%$. At high suction speeds $(0.109,0.128$, 0.147 and $0.166 \mathrm{~cm} \mathrm{~s}^{-1}$ ) the presence of particles leads to complete removal of the particle-laden fluid from the beaker. At low suction speeds $\left(0.0704\right.$ and $\left.0.0895 \mathrm{~cm} \mathrm{~s}^{-1}\right)$ the suction fraction peaks in a certain range of particle concentrations and then decreases.

(d) The cleaning property of the particle-laden siphon is here related to the screening of particles at the tube entrance.

This work was supported in part by the DOE (Engineering Research Program of the Department of Basic Engineering Sciences) and the National Science Foundation under Grant No. 0109079 (GOALI).

\section{REFERENCES}

Bailey, F. E. JR. \& Koleske, J. V. 1976 Poly (ethylene oxide). Academic Press.

Bird, R. B., Armstrong, R. C. \& Hassager, O. 1977 Dynamics of Polymeric Liquids. Fluid Mechanics, Volume 1. Wiley.

Binding, D. M. \& Walters, K. 1988 On the use of flow through a contraction in estimating the extensional viscosity of mobile polymer solutions. J. Non-Newtonian Fluid Mech. 30, 233-250.

BoONACKer, N. T. 1999 Filtration of particles suspended in Newtonian and non-Newtonian fluids. Master Thesis, Department of Aerospace Engineering and Mechanics, University of Minnesota.

Joseph, D. D. 1990 Fluid Dynamics of Viscoelastic Liquids. Springer.

LiU, Y. J. 1995 Particle motions in non-Newtonian fluids. PhD thesis, Department of Aerospace Engineering and Mechanics, University of Minnesota.

Macosko, C. W. 1994 Rheology: Principles, Measurements, and Applications. Wiley-VCH.

Matthys, E. F. 1988 Measurement of velocity for polymeric fluids by a photochromic flow visualization technique: the tubeless siphon. J. Rheol. 32, 773-788. 